\title{
A preliminary assessment of BTX pollution in urban air in the city of Lanzhou
}

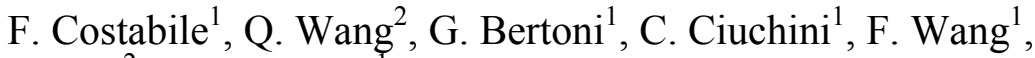 \\ L. Xue ${ }^{2} \&$ I. Allegrini ${ }^{1}$ \\ ${ }^{1}$ Institute for Atmospheric Pollution, National Research Council, \\ Rome, Italy \\ ${ }^{2}$ Environmental Research Center, Lanzhou, People's Republic of China
}

\begin{abstract}
A multi-pollutant assessment of Benzene, Toluene and Xylene was performed in the Chinese urban environment of Lanzhou city by means of diffusive samplers. The focus was upon an assessment of the interrelationships between different pollutants and different types of sampling sites. The considerable number of samples taken with the technique proposed in this work allows for the statistical treatment of data along the space coordinates. That is very useful in air quality management and control, due to the possibility to infer the statistical distribution of data and compare its parameters with reference values, such as mean, limit or standard values, etc. It was found that distributions of BTX are successfully estimated from a statistical point of view from the Normal distribution. Therefore, it was demonstrated that the passive sampling method can be used in a large-scale project for the measurements of BTX spatial variability at extremely low cost. Errors found in measurement technique are extremely small and valuable for the purposes of this work. Moreover, many of the measurement sites in this study could not be operated using conventional methods due to the difficulties in transportation, power supply, location, etc. Thus this technique allows to map big areas difficult to be reached with heavy or large instrumentation.
\end{abstract}

Keywords: air pollution, passive samplers, BTX, frequency distribution. 


\section{Introduction}

The preliminary assessment of atmospheric pollution is a very important step for the definition of locations where to deploy monitoring stations [1]. In order to perform it, diffusive sampling technique can be applied as a supplementary method to the manual, active sampling methods and automated ones. Together, the three groups of methods may form an air pollution monitoring system that is capable of providing data of high quality and very good resolution in terms of both time and space [2-4]. However, passive sampler can only integrate exposure to give a cumulative value. Hourly fluctuations in pollutant gas concentrations cannot be determined directly. At present, efforts from ecological perspective are underway to model hourly concentrations using patterns in hourly data from nearby instrumental monitors [5] as well as using climate data and altitude [6-8]. Such work could output geostatistical data that would be an improvement over just mapping seasonal averages or total accumulative exposure to a pollutant gas [9].

This paper reports the preliminary results of a bigger on-going project, "Air Quality Monitoring System and Greenhouse Gas emission Inventory", in the city of Lanzhou, P. R. China. The focus on BTX data reported in this study is valuable considering that only a few research has already been done in China relative to these pollutants.

\section{Experimental}

Analyst ${ }^{\circledR}$ passive samplers for BTX [10] have been placed in 40 locations in order to identify the spatial distribution of atmospheric pollutants. The Analyst BTX sampler is a disposal for the determination in the atmosphere of organic species (gases and vapors): Benzene, Toluene, Xylene. This device exploits molecular diffusion principles (first Fick's low) which allow a passive sampling (i.e. without pumps) in an adsorbing bed able to retain the interesting species. Sampling is simply performed by opening the disposal in the interesting atmosphere. The exposure time (which is the time between the opening and the plugging of the disposal) depends on the expected concentration of the interesting species in the atmosphere. The ambient concentrations of the organics are calculated on the basis of the time exposure, the absolute quantities of each sampled species (analytically determined) and its diffusion coefficient. For this work, the sampling time has been fixed at 1 month, Autumn-time, starting from October $15^{\text {th }}$ and ending November $15^{\text {th }}, 2005.35$ sampling locations have been selected in the urban area by fitting a geometric grid as more as possible (fig.1). 5 samplers were positioned out of the urban area, to assess regional background concentrations. In selecting the micro-scale locations of each site some indications were followed: (a) height of passive samplers from 3 to $6 \mathrm{~m}$ (e.g.: on the light poles); (b) samplers were not put in hot spots such as crossroads, traffic lights, parking; (c) sampling points were at least $25 \mathrm{~m}$ from the edge of major junctions and at least $4 \mathrm{~m}$ from the centre of the nearest traffic lane. 


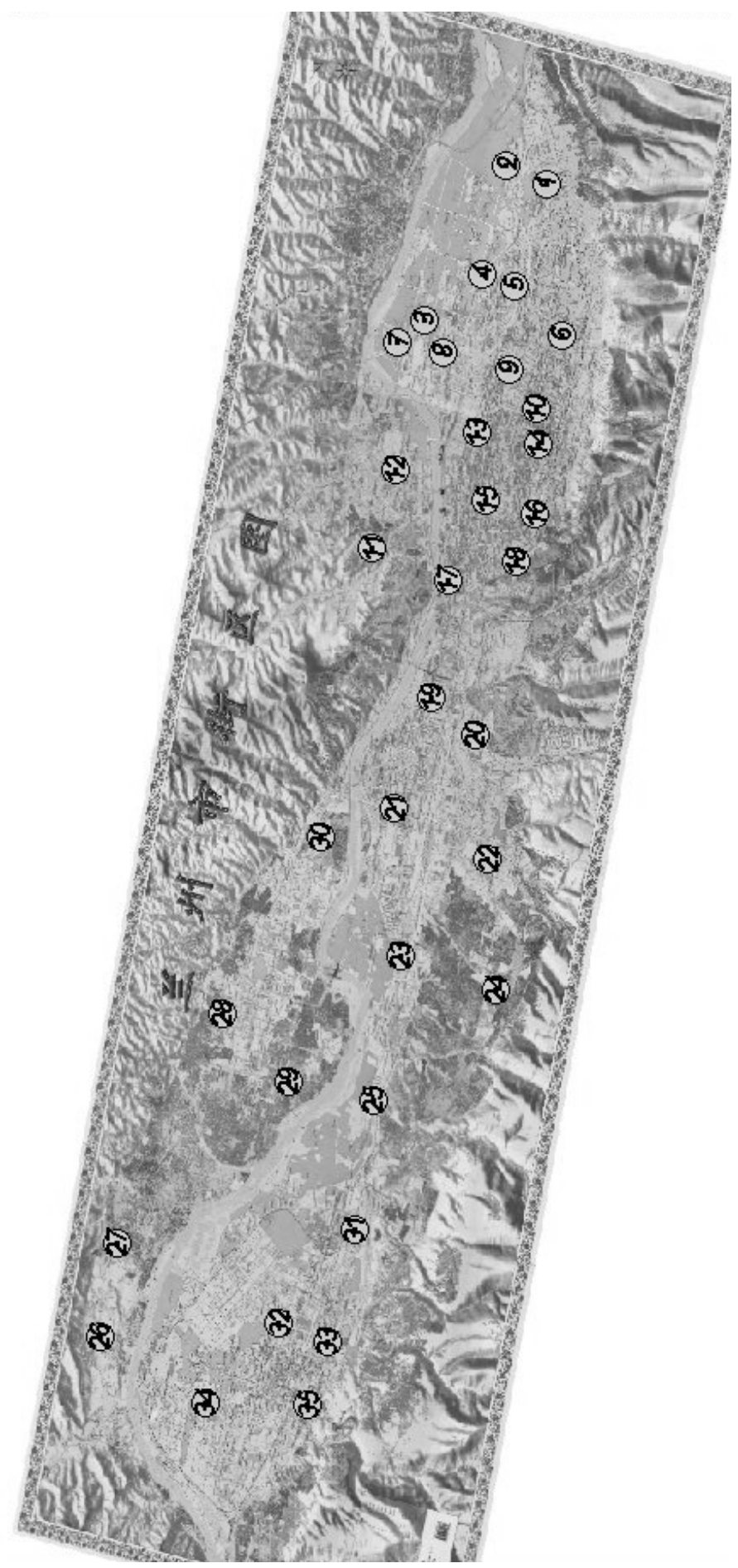

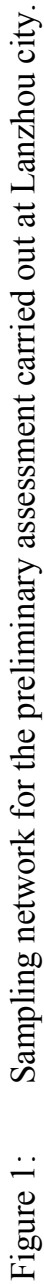

WIT Transactions on Ecology and the Environment, Vol 86, (c) 2006 WIT Press www.witpress.com, ISSN 1743-3541 (on-line) 
Quality assurance and Quality Control have been considered by duplicating 8 sampling sites among the 40 locations. Duplicated samplers were installed in order to evaluate the sampling error:

$$
\mathrm{E}=\vartheta_{1}-\vartheta_{2}
$$

where $\vartheta_{\mathrm{i}}$ is the pollutant concentration at the duplicated site $\mathrm{i}$.

Further, duplicate samples got an idea on how concentrations vary within short distances. Correlation coefficients (r) of all elements between the two sets of samples were calculated by

$$
r=\frac{\sum_{i}\left[\left(x_{i}-\bar{x}\right)\left(y_{i}-\bar{y}\right)\right]}{\sqrt{\sum_{i}\left(x_{i}-\bar{x}\right)^{2} \sum_{i}\left(y_{i}-\bar{y}\right)^{2}}}
$$

Calculations about BTX concentrations were performed by calculating the sampled volume from the experimental data of the diffusive uptake rate. In any case calculations about the ambient concentration of any interesting species may be performed by applying the following formula:

$$
C_{i}=\frac{9.62 \cdot 10^{3} \cdot m_{i}}{t \cdot D_{i}}
$$

where $C_{i}$ average ambient concentration during the sampling time. $9.62 \cdot 10^{3}$ constant of the "Analyst" sampler, $m_{i}$ mass of the $i$ species, analytically determined, $t$ exposure time, in minutes, $D_{i}$ diffusion coefficient of $i$ species in air $\left(\mathrm{cm}^{2} \cdot \mathrm{sec}^{-1}\right)$.

Atmospheric pressure does not affect calculations while ambient temperature influence the $\mathrm{D}_{i}$ values. Such influence of the temperature is usually negligible, because it is in the order of less than $2 \%$ each $10^{\circ} \mathrm{C}$. In any case, if the average temperature is significantly different from $25^{\circ} \mathrm{C}$, the previously indicated formula becomes:

$$
C_{i}=\frac{9.62 \cdot 10^{3} \cdot m_{i} \cdot \sqrt{T}}{t \cdot D_{i} \cdot \sqrt{298}}
$$

where $\mathrm{T}=$ average ambient temperature in ${ }^{\circ} \mathrm{K}$.

By applying the above mentioned formulas the resulting ambient concentration is expressed in the same units used to indicate the mass value per cubic meters: if the mass is expressed in $\mu \mathrm{g}$, you obtain $\mu \mathrm{g} \mathrm{m}^{-3}$, if the mass is expressed in $\mathrm{mg}$, you obtain $\mathrm{mg}^{-3}$ and so on.

After the experimental campaigns, the results of these techniques have been complemented by statistical studies. Sigmaplot 9.0 and Sigmastat 3.0 have been used to statistically describe the data. The frequency diagrams and histograms have usually irregular trends due to the fact that they are based on real samples. The relative data often have too low dimension to easily evidence their distribution by associating to each casual phenomenon that mathematical model as more as suitable (shape, mean value, variance, asymmetry) to the phenomenon itself. In order to overcome these problems, statistical data normality has been 
investigated by studying the maximum cumulative distance (m.c.d.) between the histogram of measured data and the Gaussian distribution curve of data.

\section{Results and discussion}

Statistical description of raw data (40 samplers) is presented in table 1.

Table 1: $\quad$ Descriptive statistics.

\begin{tabular}{|lllllllllll|}
\hline & Mean & Std.D. & Range & Max & Min & Median & $25 \%$ & $75 \%$ & Sk. & Kur. \\
\hline Benzene & 10.5 & 6.5 & 37.8 & 39.1 & 1.3 & 9.3 & 7.9 & 11.6 & 2.3 & 8.7 \\
Toluene & 15.4 & 8.0 & 39.8 & 42.5 & 2.6 & 14.9 & 10.5 & 17.7 & 1.1 & 2.4 \\
Xylene & 11.9 & 10.3 & 56.4 & 57.3 & 0.9 & 9.8 & 6.0 & 13.7 & 2.6 & 9.3 \\
\hline
\end{tabular}

The correlation coefficient $r$ of the duplicates samples showed a good correlation between the co-located samples, as shown in table 2 .

Table 2: $\quad$ Correlation coefficient $\mathrm{r}$ of duplicated samples for BTX.

\begin{tabular}{|l|lll|}
\hline \multirow{2}{*}{$\mathrm{r}$} & Benzene & Toluene & Xylene \\
\cline { 2 - 4 } & 0.985441 & 0.93542 & 0.91514 \\
\hline
\end{tabular}

Error for Benzene

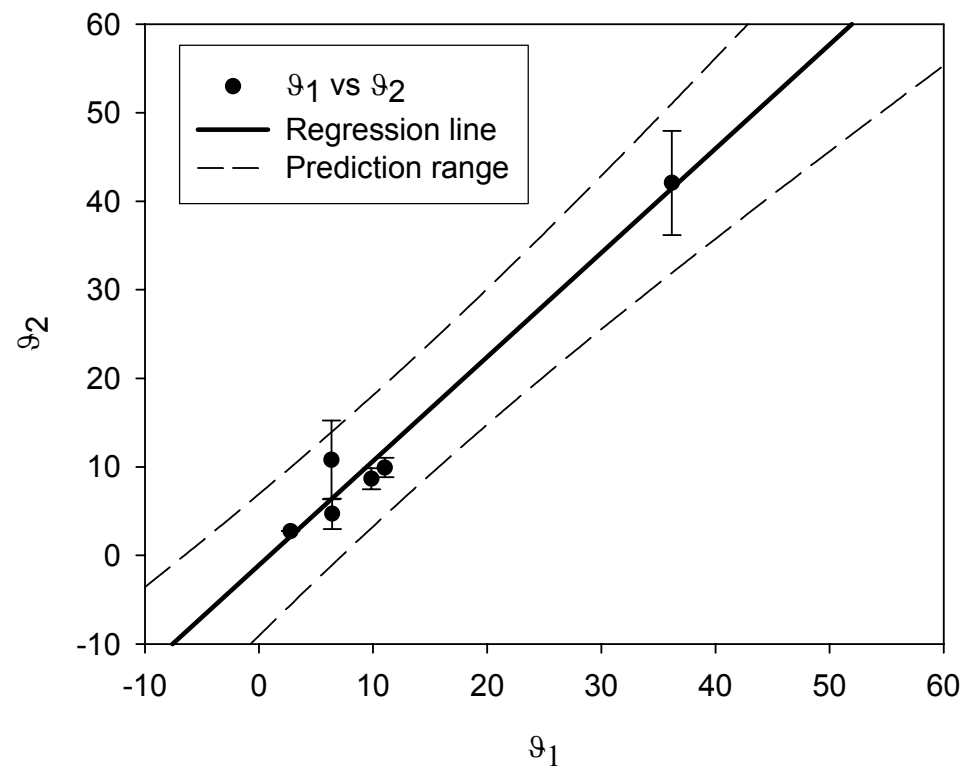

Figure 2: Scatter plot with error bars and regression of Benzene duplicated sampler $\vartheta$. The dotted line indicates the prediction range.

The sampling error was calculated for each pollutant and is shown in figures 2-4. Corresponding regression lines are shown in table 3. 


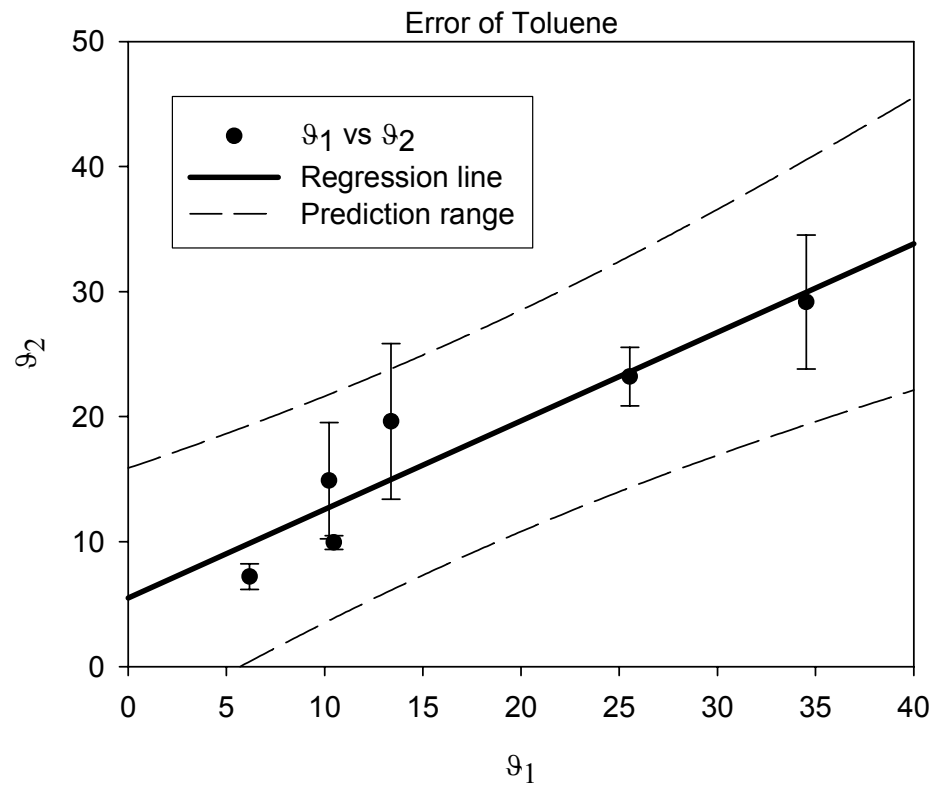

Figure 3: Scatter plot with error bars and regression of Toluene duplicated sampler $\vartheta$. The dotted line indicates the prediction range.

\section{Error for Xylene}

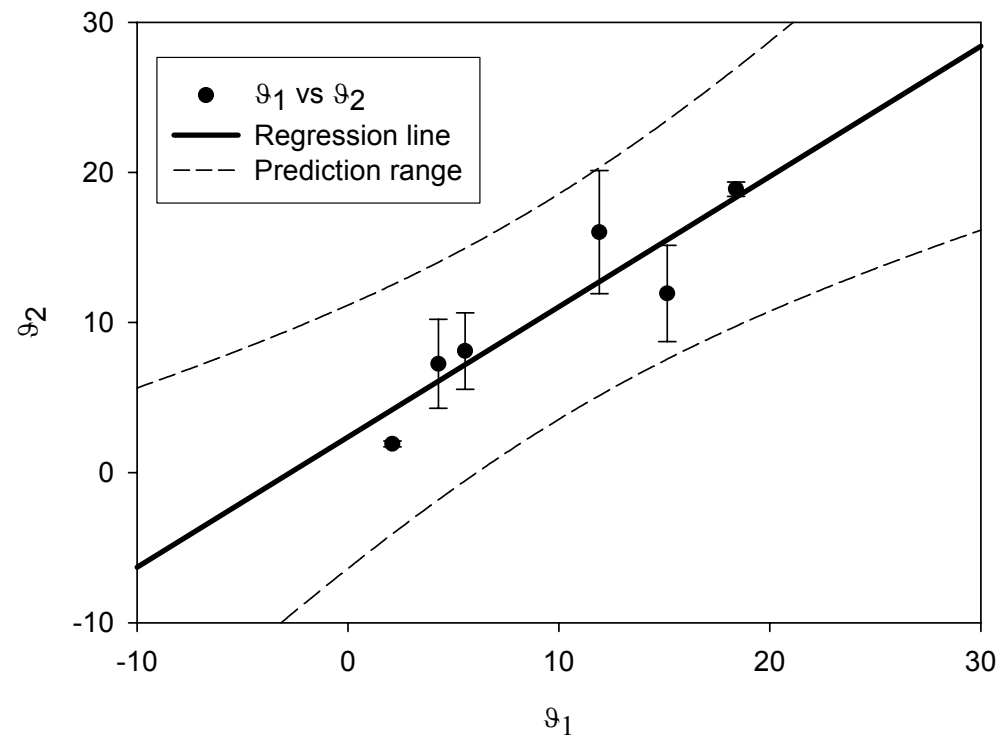

Figure 4: Scatter plot with error bars and regression of Xylene duplicated sampler $\vartheta$. The dotted line indicates the prediction range. 
Table 3: Coefficients of regression lines relative to sampling error calculated among the duplicated samples of BTX.

\begin{tabular}{|l|l|l|l|}
\hline Regression line & \multicolumn{1}{l}{ Benzene } & \multicolumn{1}{l|}{ Toluene } & \multicolumn{1}{l}{ Xylene } \\
\hline Equation & $\mathrm{Y}=-1.074+1.175 \mathrm{X}$ & $\mathrm{Y}=-5.48+0.70 \mathrm{X}$ & $\mathrm{Y}=-2.38+0.86 \mathrm{X}$ \\
\hline $\mathrm{r}^{2}$ & 0.971 & 0.875 & 0.837 \\
\hline
\end{tabular}

The angular coefficients of the BTX regression lines are close to 1 as well as the biases: that indicates the low errors of sampling found in this work suggesting a good accuracy of the measurement.

Figure 5 shows the box plot of Benzene (1), Toluene (2) and Xylene (3) data relative to all the 40 sampling locations. Benzene values are low dispersed and centred on $10 \mu \mathrm{g} / \mathrm{m}^{3}$, corresponding to the limit value set by European Union. More dispersed values were found for Xylene and Toluene, as indicated by the bigger length of the box in figure 5 , with the $75^{\text {th }}$ percentiles always less than 20 $\mu \mathrm{g} / \mathrm{m}^{3}$.

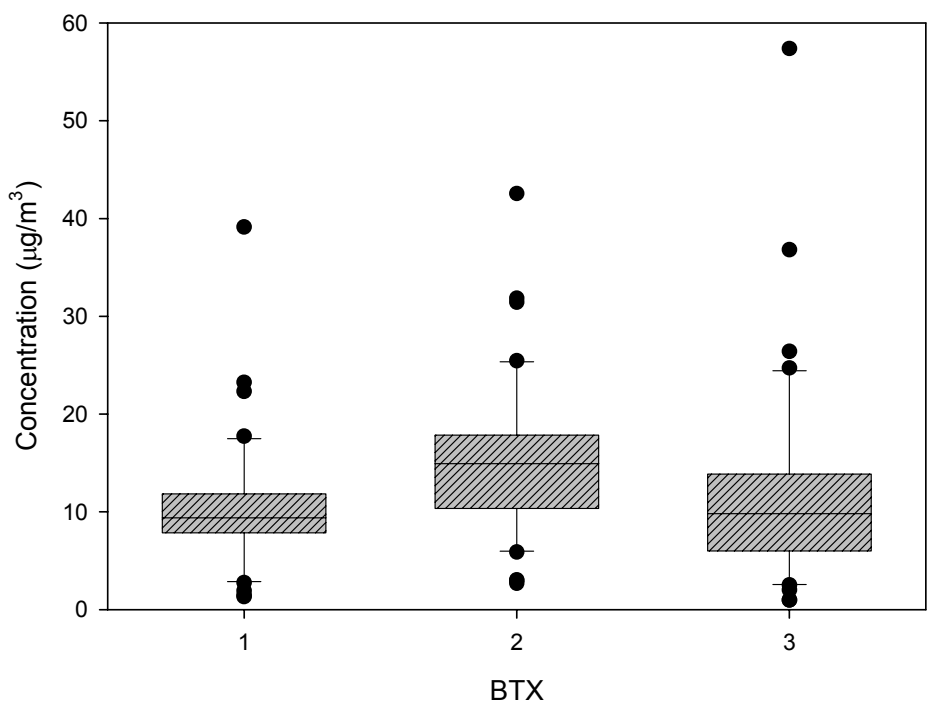

Figure 5: Box plot of BTX concentrations calculated among the 40 samplers ( $1=$ Benzene; $2=$ Toluene; $3=X y l e n e)$. The concentration is expressed in $\mu \mathrm{g} / \mathrm{m}^{3}$. Upper and lower dots indicates the outliers. Filled boxes indicates interquartile and horizontal lines show the Max and min values.

Outliers values were found. They may be expressed by the following:

Outlier $=\left\{\begin{array}{l}I F>\operatorname{Max}+1,5 \bullet I Q \\ I F<\min -1,5 \bullet I Q\end{array}\right.$, 
where (tab.1) IQ indicates the interquartile range, that is:

$$
\mathrm{IQ}=75^{\text {th }} \text { percentile- } 25^{\text {th }} \text { percentile. }
$$

The data set comprised between the maximum and minimum values (not including the outliers) were tested for statistical Normality of the frequency distribution in order to evaluate the statistical model describing it. The results are shown in table 4.

Table 4: Normality test for the BTX data (m.c.d.= maximum cumulative distance).

\begin{tabular}{|l|l|l|}
\hline Pollutant & m.c.d. & Result \\
\hline Benzene & 0.122 & Passed \\
\hline Toluene & 0.071 & Passed \\
\hline Xylene & 0.084 & Passed \\
\hline
\end{tabular}

A test that passes indicates that the data matches the pattern expected if the data was drawn from a population with a Normal distribution. That result is very important in understanding the characteristic of the spatial trend of BTX in the city of Lanzhou. Every process where the pollutant particles show a Normal distribution of the spatial trend density, satisfies the diffusion equation and thus represent such a mechanism [11]. Moreover, being the spatial data distribution Normal, mean and median values (quite same) can correctly be used to represent the concentration values of these pollutants all over the city for air quality management purposes and calculation of air pollution indexes.

\section{Conclusions}

It was demonstrated that the passive sampling method can be used in a largescale project for the measurements of BTX spatial variability at extremely low cost. Errors found in measurement technique is extremely low and valuable for the purposes of this work. Moreover, many of the measurement sites in this study could not be operated using conventional methods due to the difficulties in transportation, power supply, location, etc. Thus this technique allows to map big areas difficult to be reached with heavy or large instrumentation.

The considerable number of samples taken with the technique proposed in this work allows for the statistical treatment of data along the space coordinates. That is very useful in air quality management and control, due to the possibility to infer the statistical distribution of data and compare its parameters with reference values, such as mean, limit or standard values, etc.

The results of the on-going sampling campaigns performed all over one year (2006) of BTX measurements, as well as inorganic pollutants, will give us a comprehensive multi-pollutant, multi-seasonal assessment of air pollution in the city. In such a way it will be possible to understand reasons and causes of the pretty high concentration values measured, as well as investigate the sourcereceptor relationship of these pollutants, which was not studied in this paper. 


\section{Acknowledgements}

This study has been part of the Sino-Italian Cooperation Program For Environmental Protection-Air Quality Monitoring System City of Suzhou project. Financial support mainly from Italy Ministry of Environment and Territory (IMET) is gratefully acknowledged, as well as the policy supporting from China State Environmental Protection Administration (SEPA).

\section{References}

[1] Costabile, F., Desantis, F., Wang, F., Hong, W., Liu, F. and Allegrini, I. Representativeness of Urban Highest Polluted Zones for Sitting TrafficOriented Air Monitoring Stations in a Chinese City. JSME International journal (in press)

[2] Bower, J.S. 1992. Urban air quality monitoring - Methodology and quality assurance implications. Warren Spring Laboratory, Stevenage, UK

[3] UNEP/WHO: 1994, Passive and Active Sampling Methodologies for Measurement of Air Quality.

[4] Guidance Report on Preliminary Assessment under EC Air Quality Directives of January 1998, Project Manager Gabriel Kielland, European Environment Agency

[5] Krupa, S.V., Legge, A.H., 2000. Passive sampling of ambient, gaseous air pollutants: an assessment from ecological perspective. Environmental pollution 107, 31-45

[6] Krupa, S., Nosal, M., Peterson, D.L., 2001. Use of passive ozone (O3) samplers in vegetation effects assessment. Environmental pollution 112, 303-309

[7] Mazzali, C., Angelino, E., Gerosa, G., 2002. Ozone risk assessment and mapping in the Alps based on data from passive samplers. The scientific World 2, 1023-1035.

[8] Fraçzek, W., Bytnerowicz, A., Arbaugh, M.J.,2001. Application of the ESRI geostatistical analyst for determining the adequacy and sample size requirements of ozone distribution models in the Carpathian and Sierra Nevada mountains. In : Proceedings of the International Symposium on Passive sampling of Gaseous Air Pollutants in Ecological effects research. The scientific world 1, 836-854.

[9] Cox, R.M., 2003. The use of passive sampling to monitor forest ecposure exposure to $\mathrm{O} 3, \mathrm{NO} 2$ and $\mathrm{SO}$ : a review and some case studies. Environmental pollution 126, 301-311

[10] Bertoni, G., Tappa, R., Allegrini, I., 2001. The internal consistency of the "Analyst" diffusive sampler - a longterm field test. Chromatographia 54, 653-657

[11] Buccianti, A., Rosso, F., Vlacci, F. (Chapter 3). Metodi matematici e statistici nelle scienze della terra, ed: Liguori, Naples, pp. 204-206, 2003. 